\title{
Costo-Efectividad De Exploración De Miembros Pélvicos Para Prevenir Pie Diabético
}

\section{Reyes-Chávez Prishila Danae}

Especialista en Medicina Familiar, Unidad de Medicina Familiar, Instituto Mexicano del Seguro Social, Delegación Querétaro, México

\section{Villarreal-Ríos Enrique}

Especialista en Medicina Familiar, Unidad de Investigación Epidemiológica y en Servicios de Salud, Instituto Mexicano del Seguro Social, Delegación

Querétaro, México

\section{Martínez-Martínez Martha Leticia Velázquez-Tlapanco Jorge}

Especialista en Medicina Familiar, Docente Investigador Facultad de

Medicina, Universidad Autónoma de Querétaro, México

\section{Camacho-Calderón Nicolás}

Especialista en Pediatría y Cardiopediatría. Docente Investigador Facultad de

Medicina, Universidad Autónoma de Querétaro, México

\section{Gallardo-Vidal Lilia Susana \\ Bello-Sánchez Ma. Azucena \\ Narváez-Escobedo Miguel Ángel}

Especialista en Medicina Familiar, Unidad de Medicina Familiar, Instituto Mexicano del Seguro Social, Delegación Veracruz Sur, México

\section{Azpeitia-Castillo Nora}

Especialista en Medicina Integrada, Hospital General de Querétaro, Secretaría de Salud, Delegación Querétaro, México

\section{Abstract}

Diabetic neuropathy is a common and frequent complication of DM2. It is the main risk factor for ulcer and amputation of lower limbs. The risk of developing ulcer foot is $12-25 \%$. In the first level of care medical, history and physical examination of the patient with DM2 is performed to determine the appropriate treatment. Objective: Determine the costeffectiveness of the exploration of pelvic limbs to prevent diabetic foot. Materials and Methods: Cost-effectiveness design. Clinical records of patients with DM2. Sample calculation with the formula of percentages of two populations. Group 1, complete scan of pelvic limbs ( $n=15)$ and Group 2 
with incomplete examination of lower limbs $(n=40)$. Sociodemographic variables, health characteristics, type and number of laboratory studies requested, type and quantity of drugs prescribed were analyzed. The effectiveness was evaluated as absence of data from diabetic foot in five years follow- up. The cost of care was estimated with the profile of use and unit cost. Statistical analysis averages, percentages and cost-effectiveness. Results: The total cost in group 1 was $\$ 7,203.24$ and \$4,909.03 in 2. Group 1 , the total cost in laboratory studies was \$5,863.44, \$ 187.62 in drugs and average cost of $\$ 1,152.18$ in consultation; while in the 2 , laboratory it was $\$$ 3,596.76, \$ 186.24 medicines and average cost of consultation $\$ 1,126.03$. The effectiveness for $\$ 5,000.00$ in group 1 corresponds to $\$ 37.00$ and in the group 2 to $\$ 79.75$. Conclusions: It was no scanning showed that full pelvic limbs and timely detection of diabetic foot out cost-effective.

Keywords: Cost-effectiveness, DM2, pelvic limbs exploration, diabetic foot

\section{Resumen}

La neuropatía diabética es una complicación frecuente y precoz de la DM2. Es el principal factor de riesgo para úlcera y amputación de miembros pélvicos. El riesgo de desarrollar úlcera del pie es del 12 al 25\%. En el primer nivel de atención médica se realiza la historia clínica y exploración física del paciente con DM2 para establecer el tratamiento adecuado. Objetivo. Determinar el costo-efectividad de la exploración de miembros pélvicos para prevenir el pie diabético. Material y métodos. Estudio de costo-efectividad. Expedientes clínicos de pacientes con DM2. Cálculo de muestra con la fórmula de porcentajes para dos poblaciones. Grupo 1 con exploración completa de miembros pélvicos $(n=15)$ y grupo 2 con exploración incompleta de miembros pélvicos $(n=40)$. Las variables sociodemográficas, características de salud, tipo y número de estudios de laboratorio solicitados, tipo y cantidad de medicamentos prescritos fueron analizadas. La efectividad se evaluó como ausencia de datos de pie diabético en cinco años de seguimiento. El costo de la atención se estimó con el perfil de uso y costo unitario. Análisis estadístico con promedios, porcentajes y de costo efectividad. Resultados. El costo total en el grupo 1 fue de \$7,203.24 y en el 2 de $\$ 4,909.03$. Del grupo 1 , el costo total en estudios de laboratorio fue de $\$ 5,863.44$, en medicamentos de $\$ 187.62$ y el costo promedio en consulta de $\$ 1,152.18$; mientras que en el 2, de laboratorio fue de $\$ 3,596.76$, medicamentos de $\$ 186.24$ y costo promedio de consulta de $\$ 1,126.03$. La efectividad para $\$ 5,000.00$ en el grupo 1 corresponde a $\$ 37.00$ y en el grupo 2 , a $\$ 79.75$. Conclusiones. No se demostró que la exploración de miembros pélvicos completa y la detección oportuna de pie diabético fuera costoefectiva. 
Palabras clave: Costo-efectividad, DM 2, exploración miembros pélvicos, pie diabético

\section{Introducción}

La diabetes mellitus tipo 2 (DM2) es un problema de salud pública, en la actualidad es considerada la epidemia del siglo XXI y un reto de salud global. La Organización Mundial de la Salud estima que a nivel mundial, de 1995 a la fecha se ha triplicado la prevalencia de diabetes, con una prevalencia actual de 9.1. De acuerdo con la Federación Internacional de Diabetes; China, India, Estados Unidos, Brasil, Rusia y México son los países con mayor número de pacientes con DM2 $(1,2)$.

La DM2 es uno de los mayores problemas en salud para los sistemas de salud de Latinoamérica. El número de casos estimado para el año 2030 es mayor en nuestro país que lo pronosticado para los otros; se espera para ese año 39.9 millones de casos. La expectativa de crecimiento se basa en la alta prevalencia de obesidad e intolerancia a la glucosa. El número creciente de casos y la complejidad del tratamiento de las enfermedades crónicas han determinado un mayor número de muertes e incapacidades resultantes de la enfermedad (3).

El número de muertes atribuibles a la DM2 en el año 2011 fue de 103,300 en los hombres y 123,900 en las mujeres. La enfermedad explicó el $12.3 \%$ de las muertes totales de los adultos. El 58\% de los decesos ocurrieron en menores de 60 años. En México, la DM 2 ocupa el primer lugar en número de defunciones por año, con más de 60 mil muertes y 400,000 casos nuevos anuales. Es la primera causa de ceguera, insuficiencia renal, amputaciones no traumáticas e incapacidad laboral prematura; está entre las diez primeras causas de hospitalización y solicitud de atención médica $(4,5)$.

De las enfermedades de las extremidades inferiores, las úlceras, la neuropatía periférica, la enfermedad arterial periférica y la amputación, son dos veces más frecuentes en los pacientes con DM2; afecta al 30\% en los mayores de 40 años de edad (6).

La mayoría de las lesiones del pie diabético se inician con síntomas de neuropatía, aumento de parestesias y disminución de la sensación de dolor, lo que incrementa los traumatismos repetidos y producen mayores lesiones que asociadas a la insuficiencia vascular impiden una cicatrización oportuna (5). Del 12 al 25\% de pacientes con DM2 tienen el riesgo de desarrollar úlcera del pie, y se considera el factor de riesgo más frecuente para la amputación. Cada año se realizan más de un millón de amputaciones no traumáticas por este problema $(7,8,9)$. 
En el 2004, se registraron 65,662 egresos hospitalarios con DM2, de los cuales 12,681 se debían a hospitalizaciones por pie diabético con 5,327 amputaciones. En el 2008 se realizaron 6,223 amputaciones en instituciones sanitarias privadas. Entre el 2004 y 2005, se presentó un incremento de los egresos hospitalarios por pie diabético en 10\% y de amputaciones por diabetes en el 4\% (10).

Mendoza-Romo (2013), determinó en una Unidad de Medicina Familiar del IMSS, del estado de San Luis Potosí, México; que el costo de la complicación quirúrgica por pie diabético era de 21,000 US. Tiene un alto costo social, por el hecho de que el gasto asignado a la atención de este problema es uno de los menores (20.8 billones de dólares por año, 4.5\% del gasto mundial). El 13\% del gasto total en salud de la región es asignado a la atención de los pacientes con DM2.

Este tipo de complicaciones se ha abordado desde la perspectiva de la economía de la salud. De los diferentes tipos de evaluación económica, la de costo-efectividad es la que más se utiliza por las unidades naturales de medida que permiten la mejor toma de decisiones dentro del sistema sanitario ya que trata de comparar el costo y el efecto clínico de dos o más intervenciones alternativas, potencialmente competidoras $\mathrm{y}$, en general, mutuamente excluyentes para determinar cuál de ellas es mejor desde un punto de vista económico y clínico. Los sistemas de salud pública realizan con mayor frecuencia este tipo de estudios ya que no sólo investigan la parte clínica sino también realizan evaluaciones económicas para obtener mejores resultados al menor costo posible $(11,12,13,14)$.

Esta investigación tiene como objetivo determinar el costoefectividad de la exploración de los miembros pélvicos en el paciente con DM2.

\section{Texto principal}

Estudio de costo-efectividad. Expedientes de pacientes con DM2, pertenecientes a unidades de primer nivel de atención del Instituto Mexicano del Seguro Social, de la delegación Querétaro, México, de enero 2008 a diciembre de 2013.

Se incluyeron expedientes de pacientes con DM2, de 15 a 25 años de evolución de la enfermedad, con un mínimo de cuatro consultas médicas al año, con notas clínicas del expediente electrónico que incluyera la revisión clínica de pie. Se excluyeron los expedientes con diagnóstico de pie diabético, insuficiencia renal terminal y/o retinopatía diabética antes del $5^{\circ}$ año de seguimiento.

El tamaño de la muestra se calculó con la fórmula de porcentajes para dos poblaciones, con nivel de confianza del 95\% y poder de la prueba para una cola del 80\%, con una relación 1:2.6, grupo 1: 15 y grupo 2: 40. 
La técnica muestral fue no probabilístico, por cuota. Se analizaron las variables sociodemográficas (edad, sexo, estado civil, ocupación), características de salud (tiempo de evolución de la DM2, número de citas médicas en un año, comorbilidades, exploración física de miembros pélvicos), tipo y número de labaratorio solicitados (glucosa, biometría hemática, urea, creatinina, nitrógeno ureico o BUN (Blood Urea Nitrogen), hemoglobina glicosilada, colesterol, colesterol de alta densidad HDL, colesterol de baja densidad LDL, triglicéridos, examen general de orina, ácido úrico, depuración de creatinina en orina de 24 horas) y tipo y cantidad de medicamentos prescritos (metformina, glibenclamida, acarbosa, insulina de acción intermedia NPH, insulina rápida, complejo b, carbamazepina, pentoxifilina, ácido acetilsalicílico, dicloxacilina, metronidazol, ciprofloxacina, clindamicina).

Se conformaron dos grupos. El grupo 1, con notas de la exploración de miembros pélvicos de acuerdo a los criterios del Michigan Neuropathy Screening Instrument (apariencia del pie, ulceración, reflejo aquíleo, percepción de vibración y monofilamento). Grupo 2, sin exploración de miembros pélvicos o con datos parciales e incompletos de la exploración cuando estaban ausentes o incompletos los criterios referidos.

La efectividad fue evaluada con la ausencia de diagnóstico de pie diabético en las notas médicas del expediente electrónico a los cinco años de seguimiento.

El costo de la atención se estimó empleando el perfil de uso y el costo unitario. Para el perfil de uso del servicio se calculó por el tipo y número de ocasiones en que se utilizó en un periodo de cinco años. Los servicios fueron: Medicina familiar, laboratorio (glucosa, biometría hemática completa, urea, creatinina, BUN, hemoglobina glucosilada, colesterol, colesterol HDL, colesterol LDL, trigliceridos, EGO, ácido urico, depuración de creatinina en orina de 24 horas) y farmacia (metformina, glibenclamida, acarbosa, insulina $\mathrm{NPH}$, insulina rápida, complejo B, carbamazepina, pentoxifilina, ácido acetilsalicílico, dicloxacilina, metronidazol, ciprofloxacina o clindamicina).

Para el costo unitario se consideró el costo unitario fijo y variable. El costo unitario fijo se calculó con la técnica de tiempos y movimientos. En un primer momento por servicio se identificaron los insumos humanos, físicos y materiales; posteriormente se incorporó el gasto anual por insumo y se dividió entre el total de minutos laborados en el año; el producto se constituyó como el costo del minuto. Esta cifra se multiplicó por el total de minutos empleados en cada procedimiento y se obtuvo el costo unitario fijo.

En el costo unitario variable se consideró la técnica de microcosteo que incluye la cantidad de medicamentos y de reactivos de laboratorio. 
Se obtuvo el costo promedio anual para la consulta de medicina familiar, laboratorios y medicamentos de los años 2008, 2009, 2010, 2011 y 2012, multiplicando el uso anual de los servicios durante cada año por el costo unitario del año correspondiente, asumiendo el 3\% de inflación, en un segundo momento se obtuvo el promedio de los costos de los años estudiados.

\section{Resultados}

El promedio de edad en el grupo 1 fue de $70.86 \pm 10.11$ años, y en el 2 de $63.60 \pm 10.64$ años $(p=0.02)$, predominó en ambos grupos el sexo femenino en 93.3 y $52.5 \%(p=0.005)$.

El uso del servicio de laboratorio es más empleado en el grupo 1, ya que la biometría hemática se solicita 8.6 veces por año, comparado contra 3.55 en el grupo 2. El costo unitario de cada uno de los estudios de laboratorio fluctúa entre \$56.91 y \$124.74.

El costo promedio por tipo de estudio de laboratorio más alto es de $\$ 668.44$ y corresponde a los triglicéridos en el grupo 1, este mismo estudio reporta un costo promedio de $\$ 401.38$ en el grupo 2, en el cuadro 1 se presenta el costo promedio por tipo de estudio de laboratorio.

El costo total en estudios de laboratorio en el grupo 1 es de \$5 863.44 y en el grupo 2 de $\$ 3596.76$.

El uso de medicamentos es más utilizado en el grupo 1 que en el 2. Se reporta hasta 212 tabletas de glibenclamida vs. 164.25 tabletas en el grupo 2 .

El costo unitario de los medicamentos empleados fluctúa entre $\$ 1.71$ y $\$ 29.03$. El costo promedio por tipo de medicamento más alto es de $\$ 87.09$ que corresponde a la insulina NPH en el grupo 1 y de \$58.06 para el grupo 2.

El costo total de medicamentos solicitados en el grupo 1 fue de \$187.62 mientras que para el grupo 2.fué de \$186.24 (Cuadro 2).

El uso de la consulta es más alto en el grupo 1, incluso 35.27 veces comparado con 34.45 veces en el grupo 2. En el cuadro 3 se presenta el uso promedio anual de consulta.

El costo promedio de consulta es de $\$ 1,152.18$ pesos y corresponde al grupo 1, y de $\$ 1,126.03$ pesos en el grupo 2 .

El costo total en el grupo 1 fue de \$7,203.24, en el grupo 2 fue de $\$ 4,909.03$. En el Cuadro 4 se expresa el concentrado del costo total de laboratorio, medicamentos y consulta de acuerdo al grupo de pacientes.

La efectividad evaluada a través de la no presencia de pie diabético es de 53.30 en el grupo 1 y en el grupo 2 de 78.30. En el Cuadro 5 se representa el costo-efectividad por grupo.

La efectividad para $\$ 5,000.00$ pesos en el grupo 1 corresponde a 37.00 y en el grupo 2 a 79.75. Cuando se analiza el costo para el 100\% de 
efectividad en el grupo 1 corresponde a \$13,515.00 y en el grupo 2 a \$6,270.00 pesos (Veáse Cuadro 5).

\section{Discusión}

La educación para la salud en el autocuidado en el paciente con DM2, la identificación de neuropatía diabética y la enfermedad arterial periférica a través de anamnesis y examen físico completo y adecuado son actividades preventivas que deben realizar los médicos de primer nivel de atención; sin embargo, la exploración de los pies no se realiza por cuestiones tanto del médico tratante como del sistema de salud al asignar un tiempo limitado para otorgar la consulta médica que incluye la exploración física, prescripción farmacológica y recomendaciones de acuerdo a las circunstancias de salud que se encuentre el paciente con DM2.

La importancia de realizar la exploración de miembros pélvicos en el paciente con DM2 es de prevenir el desarrollo de pie diabético y los beneficios a corto, mediano y largo plazo para el paciente y su familia. En este sentido adquiere mayor importancia las acciones de prevención que la evaluación de costo-efectividad de esta acción médica aún cuando sea un referente para la toma de decisiones y la asignación de recursos al sector salud.

La evaluación de la efectividad realizada en este estudio es sólido al utilizar como referente la exploración de miembros pélvicos y su efecto a 5 años de evolución de la DM2; examen que tiene la ventaja de proporcionar un panorama integral al reflejar la presencia o no de neuropatía diabética. Además de estimar los costos del tratamiento y los exámenes de laboratorios requeridos para su evaluación.

Destaca que en la metodología de costo-efectividad se estimaron los costos de cada uno de los insumos requeridos en la atención médica que se otorga al paciente con DM2 en una institución de seguridad social. Esta situación parecería que los costos están en un rango económico inferior al compararse con una institución de atención médica privada, situación que no aplica por las características de la medicina privada, además de que el sector salud como otros están en un mercado de estructura libre de competencia con el otorgamiento de otros servicios de salud.

Es evidente que la atención integral del paciente con DM2 en la que se realiza la exploración de extremidades inferiores requiere de un mayor número de consultas y de exámenes de laboratorio asi como de prescripción específica por lo que los costos generados son mayores a diferencia de cuando no se realiza. Esto implica que el beneficio supera al costo de la atención médica. Este incremento en la utilización de recursos, obligadamente repercute en el costo promedio de las alternativas estudiadas, 
y se puede afirmar que esta diferencia de costos promedios es dependiente del uso, ya que el costo unitario es el mismo para las dos alternativas.

Derivado de lo expuesto, al comparar los costos promedios de ambos grupos la diferencia fue elevada por el alto impacto a nivel económico, pero no a nivel de salud.

El análisis de la efectividad en el grupo de exploración incompleta de miembros pélvicos es mayor comparado con el grupo de exploración completa. Así, la mejor relación costo- efectividad es en el grupo de pacientes con exploración incompleta en la que no se observa el beneficio de la exploración integral al no realizarse las otras medidas de educación, orientación y monitoreo regular en el paciente con DM2.

\section{Conclusión}

Con estos resultados se concluye que la exploración de miembros pélvicos y la detección oportuna de pie diabético no es costo-efectiva debido a un diagnóstico y manejo inoportuno, al menos no en las condiciones actuales de operación. Sin duda la capacitación del personal de salud es esencial en el manejo del paciente diabético y la búsqueda intencionada de complicaciones, por lo que es necesario replantear el funcionamiento, al menos así lo sugieren los resultados de la investigación realizada.

\section{References:}

Hernández-Ávila, M., Gutiérrez, J. P., \& Reynoso-Noverón, N. (2013). Diabetes mellitus in Mexico: Status of the epidemic. Salud Pública de México, 55, s129-s136.Aguilar, C., Gómez, R., \&

Jiménez-Corona, A., Aguilar-Salinas, C. A., Rojas-Martínez, R., \& Hernández-Ávila, M. (2013). Diabetes mellitus tipo 2 y frecuencia de acciones para su prevención y control. Salud Pública De México, 55, S137S143.

Guías, A. L. A. D. (2013). Sobre el Diagnóstico, Control y Tratamiento de la Diabetes Mellitus Tipo 2 con Medicina Basada en Evidencia. Revista de la ALAD, 1-142.

Arnold Rodríguez, M., Arnold Domínguez, Y., Alfonso Hernández, Y., Villar Guerra, C., \& González Calero, T. M. (2012). Pesquisaje y prevención de la diabetes mellitus tipo 2 en población de riesgo. Revista Cubana de Higiene y Epidemiología, 50(3), 380-391.

Whiting, D. R., Guariguata, L., Weil, C., \& Shaw, J. (2011). IDF diabetes atlas: global estimates of the prevalence of diabetes for 2011 and 2030. Diabetes Reserch Clinical Prac, 94(3), 311-321.

Isea, J., Viloria, J. L., Ponte, N., Carlos, I., Gómez, M., \& José, R. (2012). Complicaciones macrovasculares de la diabetes mellitus: cardíacas, 
vásculocerebrales y enfermedad arterial periférica. Rev. Venez. Endocrinol. Metab, 10(supl. 1), 96-110.

Charles, A., Andersen, M., Thomas, S., \& Roukis, D. (2007). “El pie diabético”. Surg Clin N Am, (87), 1149-1177.

Dubón Peniche, M. D. C. (2013). Pie diabético. Revista de la Facultad de Medicina (México), 56(4), 47-52.

Rincón, Y., Gil, V., Pacheco, J., Benítez, I., \& Sánchez, M. (2012). Evaluación y tratamiento del pie diabético. Rev Venez Endocrinol Metab, 10(3), 176-87.

Gómez, F. J. (2011). La diabetes tipo 2 en México: principales retos y posibles soluciones. Revista de la ALAD, 19(4), 148-161.

Carretero, L. F., García, C. I., Dalmau, J. M., \& Pibernat, M. R. (2005). Cómo entender un análisis de coste-efectividad. Piel, 20(4), 172-176.

European Scientific Journal April 2016 edition vol.12, No.12 ISSN: 1857 7881 (Print) e ISSN 18577431 http://eujournal.org/index.php/esj/article/view/7303/7023

Lenz-Alcayaga, R. (2010). Análisis de costos en evaluaciones económicas en salud: Aspectos introductorios. Revista médica de Chile, 138, 88-92.

Neri-Calero, C., \& Guadalupe, M. (2010). Costo-efectividad de SOHDi en pacientes con diabetes tipo 2 sin hipertensión. Rev Med Inst Mex Seguro Soc, 48(5), 535-538.

Mendoza-Romo, M. Á., Ramírez-Arriola, M. C., Velasco-Chávez, J. F., Nieva-de Jesús, R. N., Rodríguez-Pérez, C. V., \& Valdez-Jiménez, L. Á. (2013). Sensibilidad y especificidad de un modelo de utilidad para la detección de neuropatía diabética. Revista Médica del Instituto Mexicano del Seguro Social, 51(1), 34-41.

Cuadro 1. Costo promedio en pesos, por tipo de estudio de laboratorio en pacientes con diabetes mellitus tipo 2 con y sin exploración de pies

\begin{tabular}{c|ccccc} 
Laboratorio & \multicolumn{2}{c}{ Uso } & & \multicolumn{2}{c}{ Costo Promedio } \\
& \multicolumn{2}{c}{ Grupos } & Costo unitario & Grupos \\
\hline BHC total & 8.60 & 3.55 & 68.85 & 592.11 & 244.41 \\
EGO total & 8.60 & 3.43 & 56.91 & 489.42 & 194.91 \\
Glucosa total & 8.60 & 6.00 & 73.99 & 636.31 & 443.94 \\
Creatinina total & 8.60 & 5.98 & 73.99 & 636.31 & 442.09 \\
Urea total & 8.60 & 5.98 & 73.99 & 636.31 & 442.09 \\
Ácido úrico total & 7.33 & 6.55 & 73.99 & 542.59 & 484.63 \\
Nitrógeno ureico total & 1.80 & 0.95 & 73.99 & 133.18 & 70.19 \\
Colesterol total & 7.93 & 4.83 & 82.76 & 656.56 & 399.31 \\
Triglicéridos total & 8.08 & 4.85 & 82.76 & 668.44 & 401.38 \\
Colesterol HDL total & 1.20 & 0.59 & 82.76 & 99.31 & 48.80 \\
Colesterol LDL total & 1.20 & 0.54 & 82.76 & 99.31 & 44.56 \\
Hemoglobina glicosilada total & 5.40 & 3.05 & 124.74 & 673.59 & 380.45 \\
Total & & & & 5 & \\
& & & & 863.44 & 3596.76
\end{tabular}


Fuente: Base de datos de pacientes con diabetes tipo 2, del IMSS, delegación Querétaro, México, en el periodo del 2009 - 2013.

Cuadro 2. Costo promedio en pesos, por tipo de medicamento en pacientes con diabetes tipo 2 con y sin exploración de pies.

\begin{tabular}{c|ccccc} 
& \multicolumn{2}{|c}{ Uso } & & \multicolumn{2}{c}{ Costo Promedio } \\
Medicamentos & \multicolumn{2}{c}{ Grupos } & Costo unitario(\$) & \multicolumn{2}{c}{ Grupos } \\
& 1 & 2 & & 29.40 & 25.20 \\
Metformina total & 7 & 6 & 4.20 & 13.68 & 10.26 \\
Glibenclamida total & 8 & 6 & 1.71 & 28.80 & 43.20 \\
Acarbosa total & 2 & 3 & 14.40 & 3.51 & 3.51 \\
Ácido acetilsalicílico total & 1 & 1 & 3.51 & 0 & 2.10 \\
Complejo B total & - & 1 & 2.10 & 0 & 14.65 \\
Pentoxifilina total & - & 1 & 14.65 & 0 & 4.12 \\
Carbamazepina total & - & 1 & 4.12 & 87.09 & 58.06 \\
Insulina NPH total & 3 & 2 & 29.03 & 25.14 & 25.14 \\
Insulina acción rápida total & 1 & 1 & 25.14 & 187.62 & 186.24
\end{tabular}

Fuente: Base de datos de pacientes con diabetes tipo 2, del IMSS, delegación Querétaro, México, en el periodo del 2009 - 2013.

Cuadro 3. Uso, costo unitario y costo promedio en pesos, de la consulta en pacientes con diabetes tipo 2 con y sin exploración de pies.

\begin{tabular}{c|ccccc} 
& \multicolumn{2}{|c}{ Uso } & & \multicolumn{3}{c}{ Costo Promedio } \\
& \multicolumn{2}{c}{ Grupos } & Costo unitario (\$) & \multicolumn{2}{c}{ Grupos } \\
& 1 & 2 & & 1 & 2 \\
\hline Número total de citas & 7.05 & 6.89 & 163.43 & $1,152.18$ & $1,126.03$
\end{tabular}

Fuente: Base de datos de pacientes con diabetes tipo 2, del IMSS, delegación Querétaro, México, en el periodo del 2009 - 2013.

Cuadro 4. Costo total en pesos de laboratorio, medicamentos y consulta en pacientes con diabetes tipo 2 con y sin exploración de pies.

\begin{tabular}{c|cc} 
Tipo de servicio & \multicolumn{2}{|c}{ Costo total } \\
& 1 & Grupos \\
\hline Laboratorio Total & $5,863.44$ & $3,596.76$ \\
Medicamentos Total & 187.62 & 186.24 \\
Número de citas total & $1,152.18$ & $1,126.03$ \\
Total & $7,203.24$ & $4,909.03$
\end{tabular}

Fuente: Base de datos de pacientes con diabetes tipo 2, del IMSS, delegación Querétaro, México, en el periodo del 2009 - 2013.

Cuadro 5. Costo-efectividad por grupo de exploración

\begin{tabular}{c|ccccc} 
& \multicolumn{5}{c}{ Grupos } \\
& Costo & 1 & Efectividad & Costo & Efectividad \\
& $7,203.00$ & 53.30 & $4,909.00$ & 78.30 \\
\hline Efectividad para $\$ 5,000$ & & 37.00 & & 79.75 \\
Costo para $100 \%$ de efectividad & $13,515.00$ & & $6,270.00$ &
\end{tabular}

Fuente: Base de datos de pacientes con diabetes tipo 2, del IMSS, delegación Querétaro, México, en el periodo del 2009 - 2013. 